\title{
Contrat bancaire et monitoring : le cas de l'installation en viticulture
}

Bank contract and monitoring: the case of young farmers in the wine sector

\section{Julien Cadot}

\section{(2) OpenEdition}

Journals

\section{Édition électronique}

URL : http://journals.openedition.org/economierurale/3972

DOI : 10.4000/economierurale.3972

ISSN : 2105-2581

\section{Éditeur}

Société Française d'Économie Rurale (SFER)

\section{Édition imprimée}

Date de publication : 15 juillet 2013

Pagination : 21-39

ISSN : 0013-0559

\section{Référence électronique}

Julien Cadot, «Contrat bancaire et monitoring : le cas de l'installation en viticulture », Économie rurale

[En ligne], 336 | juillet-août 2013, mis en ligne le 15 juillet 2015, consulté le 01 mai 2019. URL : http:// journals.openedition.org/economierurale/3972 ; DOI : 10.4000/economierurale.3972 


\title{
Contrat bancaire et monitoring Le cas de l'installation en viticulture
}

\author{
Julien CADOT • Institut supérieur de gestion, Paris
}

La banque est le premier apporteur de ressources financières externes des jeunes viticulteurs. Cet article s'intéresse aux interactions entre les termes formels du contrat bancaire (montant de la dette, garanties, part de la dette à court terme) et le monitoring exercé par le banquier. L'analyse empirique montre que l'engagement ex ante de la banque, la prise de caution et la dette à court terme sont des déterminants du monitoring. De plus, la disponibilité du crédit ex post est associée à de meilleures performances (revenus plus élevés et maîtrise des risques) quand l'accompagnement par la banque implique du monitoring. Cependant, les garanties hypothécaires peuvent en réduire la qualité.

MOTS-CLÉS : monitoring bancaire, incitations, disponibilité du crédit, garanties, maturité du crédit

\section{Bank contract and monitoring: the case of young farmers in the wine sector}

Banks are the first provider of external finance to young wine-growers. This article deals with the interactions among the monitoring and the formal terms of the bank contract (credit availability, collaterals, short term debt). Our empirical tests reveal three determinants of monitoring: ex ante credit availability, personal guarantees and short term debt. Moreover, the ex post credit availability is clearly related to a better performance when there is monitoring than when there is not. However, land mortgage may reduce the monitoring effectiveness. (JEL: G32, G33, G35, L26, L25).

KEYWORDS: bank monitoring, incentives, credit availability, collaterals, debt maturity

$\mathbf{L}$ a banque est le principal apporteur -de capitaux externes des agriculteurs. Son rôle est critique au moment de l'installation, qui s'accompagne souvent d'investissements lourds, encouragés par les bonifications de prêts accordées par l'État aux jeunes agriculteurs. Dans ce contexte, l'accompagnement des « jeunes agriculteurs » par la banque, via les contrats mis en place et le suivi des entrepreneurs (monitoring), est un déterminant essentiel des conditions de l'installation. Cet article traite de deux aspects de l'accompagnement bancaire : le lien entre les contrats accordés par la banque et le monitoring bancaire, et les effets incitatifs des termes du contrat dans le cadre d'un accompagnement avec plus ou moins de monitoring.
Dans notre échantillon, qui comprend 272 cas d'installations réalisées entre 1998 et 2003, les jeunes agriculteurs investissent près de 10000 euros par hectare (pour une moyenne de 15 ha, très variable selon les régions viticoles), pour 11000 euros de chiffre d'affaires par hectare et en s'endettant pour près de 8000 euros par hectare. Le banquier est ici non seulement le premier apporteur externe de ressources financières aux viticulteurs mais son apport dépasse très largement l'autofinancement.

Pourtant, l'asymétrie d'information ex ante entre le banquier et le jeune viticulteur est forte. L'installation implique généralement des changements structurels en termes d'outil de production, d'orientation managériale et de structure 
financière qui font perdre toute pertinence aux notes attribuées par les logiciels de scoring. Les dossiers de financement se réfèrent logiquement à l'étude prévisionnelle d'installation plutôt qu'à l'historique de l'exploitation. Le problème d'anti-sélection est d'autant plus prononcé que les prêts bonifiés, largement accordés dans le cadre de l'installation, encouragent tous les entrepreneurs, même les plus risqués, à s'endetter pour investir. De plus, le banquier est confronté à une asymétrie d'information ex post. Il n'est pas à l'abri des formes subreptices d'aléas moraux tels que des prélèvements trop élevés de la part de l'entrepreneur au détriment de la sécurité financière de l'entreprise ou encore des investissements surdimensionnés en matériel quand l'entrepreneur surpondère les avantages en termes d'ergonomie et de gains de temps par rapport au risque financier associé à la dépense. Autrement dit, l'asymétrie d'information entre le banquier et l'entrepreneur est forte, ce qui, en théorie, devrait impliquer un dysfonctionnement du marché (Akerlof, 1970) et plus spécifiquement, du rationnement de crédit (Stiglitz, Weiss, 1981), ce qui nous amène à nous interroger sur le rôle du contrat entre le banquier et le viticulteur dans la résolution (ou l'atténuation) du problème informationnel.

Le problème peut être posé sous la forme d'une relation principal/agent, où le principal serait le banquier, qui confie son argent à un agent, qui est donc l'entrepreneur. Le principal a deux solutions pour atténuer les problèmes informationnels (Milgrom, Roberts, 1992) :

- Le monitoring : la banque estime la capacité de remboursement future par un suivi régulier de l'entreprise et de l'entrepreneur, qui est en partie déterminé par certaines composantes du contrat bancaire - la dette à court terme est une façon d'imposer un suivi (Fama, 1985 ; Jensen 1986), et les garanties de s'informer sur la nature des actifs de l'entreprise (Ono, Uesugi, 2005 ; Chakraborty, Hu, 2006).

- Un contrat incitatif : le banquier peut établir un contrat incitant les entrepreneurs à révéler de l'information sur leurs compétences ou à agir dans les intérêts de la banque, par de la dette à court terme (Stiglitz, Weiss, 1983 ; Flannery, 1986 ; Aghion, Bolton, 1992 ; Bolton, Sharfstein, 1990) ou par des garanties (Bester, 1985 ; Boot, Thakor 1994).

Le monitoring peut être considéré comme un des termes du contrat - le banquier décide du degré de suivi nécessaire à l'accompagnement de l'entrepreneur - mais a ceci de particulier qu'il est fortement déterminé par les autres termes du contrat tels la structure de la dette (long terme/court terme) ou les garanties, d'où une première question sur le rôle des termes du contrat bancaire dans le suivi de l'entrepreneur par le banquier. Autrement dit, dans quelle mesure déterminent-ils le monitoring ?

La relation entre les termes du contrat et le monitoring ne se limite pas à celle de causalité. En effet, les modèles principal/ agent démontrant les propriétés incitatives des différents termes du contrat bancaire ne présentent pas toujours la même structure. Il peut y avoir renégociation (dans le modèle d'Aghion, Bolton, 1992) ou non (modèles de Flannery, 1986 ; Bester, 1985 ) et l'information révélée au cours de la première période peut être complète (par exemple Bolton, Sharfstein, 1990) ou incomplète (Aghion, Bolton, 1992 ; Manove et al., 2001). Ces différences reposent essentiellement sur l'acquisition d'information par le principal, c'est-à-dire sur la réalisation d'un monitoring par le banquier. Se pose donc la question de l'effet du monitoring sur les propriétés incitatives des termes du contrat.

Le monitoring est un élément structurant du contrat bancaire : sa réalisation et son intensité sont en partie déterminées par les 
autres termes du contrat et il a un effet sur leurs propriétés incitatives. De ce fait, notre recherche s'articule en deux temps. Nous mettons d'abord en évidence le rôle des termes du contrat bancaire sur la réalisation ou non du monitoring. Ensuite, nous étudions leurs propriétés incitatives en fonction du monitoring réalisé par le banquier.

Notre travail empirique s'appuie sur une base de données originale construite en partenariat avec huit caisses régionales du Crédit Agricole comprenant les principales régions viticoles (hormis Bourgogne et Champagne) et Crédit Agricole SA. Il est donc difficile de tirer des enseignements généralisables au financement de l'agriculture par la banque, tant les pratiques observées sont spécifiques à l'institution qu'est le Crédit Agricole. Toutefois, le Crédit Agricole détient près de $80 \%$ des parts de marché de l'installation en agriculture $^{1}$. De ce fait, l'observation des pratiques de financement par le Crédit Agricole contribue utilement à la compréhension des problématiques financières de l'installation en France.

L'article est organisé en quatre parties : la revue de littérature revient sur le lien entre les termes du contrat et le monitoring dans le cadre de la relation banque/ PME puis sur les propriétés incitatives des termes du contrat bancaire en différenciant les modèles postulant un contrat avec monitoring ou non ; nous présentons ensuite les données, les variables et les statistiques descriptives. Dans la troisième partie, nous

1. Tout du moins, des installations aidées, c'està-dire éligibles à des aides de l'État telles qu'une subvention de trésorerie et la bonification de prêts. Ce chiffre résulte d'une évaluation interne de trois des caisses régionales partenaires de la recherche. D'autre part, la Cour des Comptes (2007) évalue à $85 \%$ la part des prêts bonifiés distribués par le Crédit Agricole. La majorité de ces prêts sont attribués dans le cadre de l'installation. Ce chiffre devrait donc être assez proche de la part de marché du Crédit Agricole sur l'installation en agriculture en général. réalisons une régression logistique où le monitoring est la variable expliquée et les termes du contrat les variables explicatives pour caractériser le rôle des termes du contrat bancaire dans le monitoring. Dans la quatrième partie, nous nous intéressons aux propriétés incitatives des termes des contrats et plus particulièrement à l'effet du monitoring sur ces propriétés par l'identification de leurs interactions. Les variables expliquées sont ici la défaillance et le revenu prélevé par les entrepreneurs sur leurs comptes professionnels.

\section{Revue de littérature}

\section{Relation ambiguë entre monitoring et contrat bancaire}

Pour Barry et Robison (2001), le monitoring bancaire est une question critique du financement des entreprises agricoles du fait de l'intensité capitalistique et de la volatilité des flux de trésorerie propre au secteur (risque « naturel » et loi de King). Il s'agit de comprendre comment le banquier obtient suffisamment d'information sur les PME pour leur proposer un financement adapté alors même que ces entreprises ne disposent pas des ressources pour produire une information formalisée sur sa situation financière et ses capacités de remboursement futures.

Pour Petersen et Rajan (1994), l'information est partagée au cours des nombreux échanges formels et informels entre le banquier et l'entrepreneur, autrement dit, via l'établissement d'une relation bancaire. Des auteurs tels que Petersen et Rajan (1994), Berger et Udell (1995), Cole (1998), Degryse et Van Cayseele (2000) et Chakraborty et $\mathrm{Hu}$ (2006) se sont interrogés sur l'impact d'une relation bancaire plus ou moins forte sur les termes du contrat (taux d'intérêt, disponibilité du crédit ou garanties). Les résultats parfois 
contradictoires ont amené les auteurs à supposer un rôle des termes du contrat dans la relation bancaire. Par exemple, Chakraborty et $\mathrm{Hu}$ (2006) s'interrogent sur le lien entre garanties utilisées et processus d'échange d'information. Ono et Uesugi (2005) ou Kano et al. (2010) proposent d'observer la relation de causalité entre les garanties et le monitoring, ouvrant ainsi la voie à l'étude du rôle des termes du contrat dans le monitoring bancaire.

\section{Les termes du contrat comme déterminants du monitoring}

L'enjeu de la négociation entre les jeunes viticulteurs et le banquier porte sur le montant de la dette à long terme, les garanties et le montant et la nature de la dette à court terme. Pour une part essentielle de la dette, le taux d'intérêt est fixé du fait des bonifications accordées par l'État dans le cadre de l'aide à l'installation. De plus, aucun covenant n'est formalisé (le banquier ne conditionne pas le financement à l'évolution d'indicateurs comptables... tout du moins, ce n'est pas écrit dans le contrat).

L'entrepreneur et le banquier s' accordent d'abord sur le montant de la dette à long terme. Autrement dit, la disponibilité du crédit est le premier élément du contrat à être négocié. Nous différencions ici la disponibilité du crédit ex ante, mesurée par le rapport entre le montant de la dette à long terme formalisée dans le plan prévisionnel d'installation et l'investissement ${ }^{2}$, et la disponibilité du crédit ex post, mesurée par le rapport entre la dette prévisionnelle et l'encours effectif constaté sur les comptes bancaires. Une forte disponibilité du crédit ex ante devrait être associée à

2. C'est un ratio de disponibilité car les entrepreneurs ont intérêt à maximiser la dette à long terme du fait de la bonification d'une part essentielle de ces prêts et de la nécessité de constituer une trésorerie pour couvrir les risques climatiques, de prix ou de méventes. du monitoring : la banque prend plus de risque, et la gestion de ce risque implique un suivi. De même, une forte disponibilité du crédit ex post implique du monitoring : la banque accepte une prise de risque supérieure à ce qu'il était convenu initialement, ce qui de fait nécessite une analyse de la situation de l'entreprise et un suivi.

La relation entre garanties et monitoring est plus ambiguë. Les garanties sont souvent supposées réduire l'effort de monitoring par le banquier, ce que formalisent Manove et al. (2001). Cependant, pour Rajan et Winton (1995), les garanties peuvent inciter les banquiers à un effort de monitoring car elles s'apparentent à un droit de propriété sur le projet de l'entrepreneur. Ono et Uesugi (2005) montrent que, dans le cas japonais, les garanties ont été prises par les banquiers dans le but d'acquérir de l'information sur les entreprises.

Plus spécifiquement, chaque type de garanties a ses propres propriétés informationnelles (Berger, Udell, 1995 ; Chakraborty, Hu, 2006 ; Ono, Uesugi, 2005). Dans le cas des entreprises viticoles, les nantissements ou la prise de caution informent sur la nature des actifs, les ressources personnelles, voire la confiance des personnes qui se portent caution en la réussite de l'entrepreneur - information intéressante quand ce sont les cédants de l'exploitation qui se portent caution pour le repreneur. L'évaluation des garanties hypothécaires se réfère à des données du marché local plutôt qu'à une analyse spécifique de l'entreprise. Par conséquent, les cautions et les nantissements devraient apparaître comme des déterminants du monitoring bancaire mais pas les garanties hypothécaires.

La dette à court terme renouvelable implique une renégociation, ce qui implique nécessairement un effort de monitoring (Fama, 1986). De plus, la capacité de rembourser la dette à court terme est également une information en soi (Flannery, 1986 ; Bolton, Sharfstein, 1990). A contrario, le 
financement de la trésorerie par le découvert évite la renégociation tout en plafonnant le montant de l'engagement. Ainsi, la dette à court terme renouvelable devrait être un déterminant du monitoring tandis que le découvert serait privilégié quand le banquier veut éviter les coûts de renégociation $^{3}$.

Ce premier développement nous amène à formuler une hypothèse générale : H1, les termes du contrat déterminent le monitoring bancaire que nous déclinons en trois hypothèses testables :

- H1a, la disponibilité du crédit ;

- H1b, les cautions et les nantissements ;

- H1c, et la dette à court terme ; sont des déterminants du monitoring.

\section{Propriétés incitatives des termes du contrat}

La renégociation des financements peut atténuer le risque d'aléa moral à la fois sur les investissements entrepris (Aghion, Bolton, 1992) et le revenu prélevé par l'entrepreneur sur l'entreprise (Bolton, Sharfstein, 1990). Les entrepreneurs anticipant une renégociation de la dette devraient minimiser les risques de défaillance. Ainsi, la disponibilité du crédit ex post devrait être associée à de meilleures performances ex ante mais également ex post puisque les financements supplémentaires sont accordés à la faveur d'un constat de bonnes performances et en plus relaxent les contraintes de trésorerie, et de revenus. En outre, la renégociation est indissociable d'une forme plus ou moins forte de monitoring (voir pour une formalisation générale du signal impliquant renégociation Aghion et Bolton, 1992). Par conséquent, la relation

3. Cette dernière hypothèse contredit Berger et Udell (1995) ou Chakraborty et $\mathrm{Hu}$ (2006) pour qui les lignes de crédit sont associées à un financement relationnel justement parce que leur utilisation est à la discrétion de l'entrepreneur. positive entre disponibilité du crédit ex post et performances financières (indicateurs d'incitation à l'effort et à un choix raisonné d'investissements) devrait être significativement plus forte dans le cadre d'un contrat avec monitoring.

La dette à court terme est un moyen, pour le banquier, de provoquer une renégociation. Elle devrait inciter les entrepreneurs à la performance (au moins du point de vue du banquier) puisque de celle-ci dépendra la disponibilité des financements futurs (Aghion, Bolton, 1992). C'est également un signal potentiel que les entrepreneurs les moins risqués peuvent utiliser pour se distinguer auprès du banquier (Flannery, 1986). Enfin, la dette à court terme peut être un moyen de discipliner le dirigeant en l'empêchant de privilégier sa consommation immédiate ou la réalisation de projets hasardeux, conformément à la théorie des free cash flows (Jensen, 1986). Pour ces trois raisons, les entreprises financées au moins en partie par de la dette à court terme devraient être les plus performantes, ce qui est d'ailleurs contradictoire avec l'approche financière « classique » préconisant l'ajustement des maturités des investissements et des dettes.

Les garanties peuvent être utilisées par les entrepreneurs pour signaler la qualité de leur projet (Bester, 1985). Quand le problème d'information se situe ex post, le banquier peut utiliser les garanties afin de réduire le risque d'aléa moral de la part de l'entrepreneur (Boot, Thakor, 1994). L'effet des garanties sur l'aléa moral devrait être d'autant plus marqué que le contrat ne comporte pas de monitoring, qui atténue déjà le risque d'aléa moral. A contrario, dans le cadre d'un contrat avec monitoring, les garanties sont susceptibles de réduire la vigilance du banquier de sorte que les entrepreneurs sont plus enclins au surinvestissement ou à un prélèvement excessif quand des garanties couvrent une partie de la dette (Manove et al., 2001). 
Cette brève revue de littérature nous amène à considérer que : H2, le monitoring détermine les propriétés incitatives des termes du contrat bancaire.

Nous déclinons $\mathrm{H} 2$ en quatre hypothèses testables :

- H2a : la disponibilité du crédit ex post est un facteur clé de la performance financière des entreprises financées via un contrat avec monitoring (effet incitatif cumulé au cercle vertueux, « la performance rend disponible les financements qui permettent d'obtenir une meilleure performance qui...») ;

- $\mathrm{H} 2 \mathrm{~b}$ : la dette à court terme incite les entrepreneurs à la prudence en termes d'investissements et de prélèvements (effet disciplinaire) ;

- H2c : les garanties incitent les entrepreneurs à la prudence dans le cadre d'un contrat sans monitoring (effet disciplinaire) ;

- H2d : les garanties réduisent la qualité du monitoring bancaire (lazy-bank effect).

\section{Les données}

\section{L'échantillon}

Les données compilent des données technico-économiques, comptables et bancaires sur 272 cas d'installations « aidées » réalisées entre 1998 et 2003. Elles ont été collectées grâce à un partenariat avec le Crédit Agricole SA qui a donné lieu à une analyse financière et bancaire d'un échantillon de «jeunes viticulteurs » délivrée au service Agroalimentaire. Notre base de données comporte notamment une information très détaillée sur les données bancaires : les flux sur les comptes, le montant des prêts, les garanties utilisées, l'épargne et les retards de remboursement.

\section{Les variables}

Les comptes professionnels et personnels sont très souvent séparés, ce qui permet d'observer la somme des flux entrants annuels sur le compte personnel qui, avec quelques limites, constitue un indicateur du revenu des agriculteurs ${ }^{4}$. Les garanties ont été classées en trois catégories : les garanties hypothécaires, personnelles et les nantissements. Affectée à différents prêts à long terme, l'information conservée donne donc le montant couvert par les garanties pour chacune de ces catégories, ce qui permet d'établir des ratios de couverture selon le type de garanties concernées. Les financements à court terme sont détaillés : nous avons reporté le montant des découverts autorisés et des encours à court terme. Il est possible de détailler la structure du financement bancaire : dette à long terme, à court terme (moins d'un an) et découverts autorisés. Nous avons pu également constater la présence de retards de remboursement sur les comptes professionnels, ce qui indique des difficultés de trésorerie. Enfin, à partir des comptabilités prévisionnelles, qui rendent compte des projets d'investissement et de financement, nous avons construit un indicateur de la disponibilité du crédit en faisant le rapport de la dette effectivement contractée (encours restant dû sur les prêts à long terme) sur la dette prévisionnelle (dette à moyen et long terme $)^{5}$. Si cet indicateur est supérieur à 1 , l'entrepreneur a obtenu plus de crédits que ce qui était initialement prévu avec le banquier. S'il est inférieur à 1 , le montant des financements accordés initialement a été restreint.

Enfin, nous avons pu constater que les dossiers de suivi des entrepreneurs comportent plus ou moins d'information.

4. Nous observons une moyenne de 26000 euros et une médiane de 17000 euros, ce qui correspond à ce qu'observent Chassard et Chevalier (2007), un revenu médian supérieur à 20000 euros pour la viticulture d'appellation et 15000 euros pour les autres. 5. Le montant prévisionnel est observable dans l'étude prévisionnelle, la plupart du temps discutée avec le banquier. 
L'acquisition et la conservation des données comptables dans le dossier ne concernent qu'un peu plus de la moitié des entrepreneurs de l'échantillon, ce que nous considérons comme un critère révélateur de l'effort de monitoring bancaire. Cet indicateur n'est pas parfait mais a l'avantage d'être une approche directe du monitoring, là où les auteurs ont utilisé la durée de la relation bancaire (Petersen, Rajan, 1994 ; Berger, Allen, $1995, \ldots)$ puis le nombre de services bancaires associés au contrat de prêt (Degryse, Van Cayseele, 2000 ; Chakraborthy, $\mathrm{Hu}, 2006, \ldots$ ) et la construction d'indice composite (Kano et al., 2010). Elsas (2005) fait figure d'exception en adoptant une approche déclarative (les chargés d'affaires désignent les entreprises avec lesquelles ils considèrent avoir établi une relation bancaire). Il s'appuie sur une spécificité du contexte allemand où la notion d'hausbank est établie pour l'ensemble des acteurs.

D'autre part, considérer l'attention portée aux documents comptables pour caractériser le monitoring est discutable. En effet, Stein (2002) et Petersen (2004) ont contribué à la théorie de la relation bancaire en définissant les concepts d'information soft (non formalisée et spécifique au contexte de production) et d'information hard (formalisée et transférable). La dimension relationnelle du contrat bancaire reposerait sur la capacité à produire de l'information soft ce qui est a priori plus adapté au financement des PME que le recours à un financement basé sur de l'information exclusivement financière et non spécifique. Dans cette perspective, Berger et al. (2005) se sont notamment posé la question de l'impact du recours aux logiciels de scoring pour le financement des PME. Un des risques supposés est que la substitution d'une relation bancaire par un processus de décision automatisé et basé sur un traitement d'information hard aboutisse à une dégradation des conditions de financement des PME. L'étude montre que le recours à ces logiciels ne modifie pas les pratiques des banques. Plus récemment, Berger et al. (2009) montrent que l'utilisation de ces logiciels a un impact positif sur le volume de prêts accordés. Ainsi, loin de se substituer à la dimension relationnelle du contrat bancaire, les processus d'acquisition d'information hard sont un complément qui permet d'augmenter le volume d'activité. Notre approche du monitoring se situe dans cette perspective. Obtenir et conserver les données comptables révèlent plutôt un effort de monitoring qu'une volonté de simplifier la prise de décision.

\section{Statistiques descriptives}

Les principales variables utilisées sont décrites dans le tableau 1 pour l'ensemble de l'échantillon. Le chiffre d'affaires moyen des entreprises de l'échantillon peut paraître faible (156 000 euros) mais l'activité agricole présente un taux de valeur ajoutée plutôt élevé relativement aux entreprises des secteurs industriels ou de négoce. Le revenu moyen (flux entrant sur le compte personnel) est de 26000 euros. $17 \%$ des entrepreneurs présentent un retard de remboursement. Ce taux assez élevé reflète sans doute la conjoncture défavorable au moment de la collecte des données (année 2005), pic d'une crise qui a touché toutes les formes de viticulture.

Certains contrats $(28 \%)$ comportent des garanties hypothécaires. Le taux de couverture moyen est de $58 \%$. Les cautions sont présentes dans $60 \%$ des contrats, pour lesquels le taux de couverture moyen est de $57 \%$. Les écarts-types sur les taux de couverture sont assez élevés, ce qui reflète la diversité des pratiques dans l'utilisation des garanties. Les nantissements sont assez fréquemment utilisés puisque présents dans $40 \%$ des contrats. En revanche, le taux de couverture par ce type de garantie est plutôt faible, en moyenne de $30 \%$ pour les contrats en comportant. 
Tableau 1. Les données

\begin{tabular}{|c|c|c|c|c|c|c|}
\hline Variable & $\begin{array}{l}\text { Abréviation } \\
\text { (tableaux } \\
\text { suivants) }\end{array}$ & Obs & Mean & Std. Dev. & Min. & Max. \\
\hline CA (millier d'euros) & & 258 & 155,933 & 178,134 & 0,000 & 1202,830 \\
\hline Revenu (millier d'euros) & & 185 & 25,862 & 27,164 & 0,000 & 174,828 \\
\hline $\begin{array}{l}\text { Retard de remboursement } \\
\text { (oui }=1 \text {, non }=0 \text { ) }\end{array}$ & & 272 & 0,17 & 0,38 & 0 & 1 \\
\hline $\begin{array}{l}\text { Garanties hypothécaires } 1 \\
\text { (taux de couverture en \%) }\end{array}$ & Hyp. & 254 & 16,52 & 29,17 & 0 & 100 \\
\hline $\begin{array}{l}\text { Garanties hypothécaires } 2 \\
\text { (oui }=1, \text { non }=0 \text { ) }\end{array}$ & & 272 & 0,28 & 0,45 & 0 & 1 \\
\hline $\begin{array}{l}\text { Garanties hypothécaires } 3 \\
\text { (taux de couvertures si utilisation) }\end{array}$ & & 72 & 58,26 & 23,80 & 5,74 & 100 \\
\hline Cautions 1 (taux de couverture en \%) & Caut. & 254 & 35,86 & 37,31 & 0 & 100 \\
\hline Cautions 2 (oui = 1 , non = 0 ) & & 272 & 0,60 & 0,49 & 0 & 1 \\
\hline $\begin{array}{l}\text { Cautions } 3 \\
\text { (taux de couverture si utilisation) }\end{array}$ & & 160 & 56,93 & 31,74 & 2,67 & 100 \\
\hline $\begin{array}{l}\text { Nantissements } 1 \\
\text { (taux de couverture en \%) }\end{array}$ & Nant. & 254 & 0,13 & 0,24 & 0 & 1 \\
\hline Nantissements 2 (oui = 1, non = 0 ) & & 272 & 0,40 & 0,49 & 0 & 1 \\
\hline $\begin{array}{l}\text { Nantissements } 3 \\
\text { (taux de couverture si utilisation) }\end{array}$ & & 106 & 31,43 & 28,35 & 0,30 & 100 \\
\hline $\begin{array}{l}\text { Dette à long et moyen terme } \\
\text { sur chiffre d'affaires (en \%) }\end{array}$ & DMLT/flux & 228 & 92,30 & 117,18 & 0,00 & 773,43 \\
\hline Disponibilité du crédit ex ante (en \%) & Disp. ex ante & 262 & 67 & 29 & 21 & 117 \\
\hline Disponibilité du crédit ex post (en \%) & Disp. ex post & 250 & 96,69 & 72,98 & 9,70 & 249,00 \\
\hline $\begin{array}{l}\text { Part du crédit à court terme } \\
\text { dans l'endettement (en \%) }\end{array}$ & DCT & 250 & 7,71 & 17,57 & 0 & 100 \\
\hline Crédit à court terme (oui $=1$, non $=0$ ) & & 250 & 0,28 & 0,45 & 0 & 1 \\
\hline $\begin{array}{l}\text { Part du crédit à court terme dans } \\
\text { l'endettement si utilisation (en \%) }\end{array}$ & & 69 & 27,90 & 23,63 & 1,64 & 100 \\
\hline $\begin{array}{l}\text { Part de l'ouverture de crédit dans } \\
\text { l'endettement (en \%) }\end{array}$ & OC & 252 & 8,86 & 17,27 & 0 & 100 \\
\hline Ouverture de crédit (oui = 1, non = 0 ) & & 252 & 0,54 & 0,50 & 0 & 1 \\
\hline $\begin{array}{l}\text { Part de l'ouverture de crédit dans } \\
\text { l'endettement si utilisation (en \%) }\end{array}$ & & 136 & 16,38 & 20,75 & 1,22 & 100 \\
\hline Durée de la relation bancaire & Durée & 257 & 7,67 & 5,75 & 1 & 28 \\
\hline
\end{tabular}

Source : l'auteur.

L'endettement représente en moyenne $92 \%$ du chiffre d'affaires de chaque entreprise mais l'écart-type est important. Pour certaines, l'endettement représente plus de sept fois le chiffre d'affaires. La disponibilité du crédit est en moyenne de $100 \%$, ce qui est logique. Toutefois, il faut remarquer l'importance de l'écart-type qui rend compte d'une très grande hétérogénéité des situations. 
La dette à court terme (il s'agit dans de nombreux cas d'un crédit campagne) n'est utilisée que par $28 \%$ des entrepreneurs et représente en moyenne $28 \%$ de la dette. L'ouverture de crédit est utilisée par beaucoup plus d'entrepreneurs (54\%) mais reste un moyen de financement complémentaire des autres types de crédit : il représente en moyenne $16 \%$ du total des dettes.

Enfin, la durée de la relation bancaire, construite à partir de la différence entre l'année 2005 (date de collecte des données) et celle de l'ouverture du premier comptecourant personnel avec la banque, montre que les entrepreneurs sont très souvent les clients personnels de la banque dans laquelle ils s'endettent, ce qui implique une relation d'une durée plutôt longue, en moyenne de 8 ans.

Tous les dispositifs contractuels à disposition du banquier ne sont pas forcément utilisés. Les garanties hypothécaires, ainsi que la dette à court terme, concernent moins d'un tiers des entrepreneurs tandis que les cautions et l'ouverture de crédit sont présentes dans plus de la moitié des contrats. De plus, l'écart-type élevé sur la disponibilité du crédit ex post montre que l'étude prévisionnelle ou le business plan ne détermine pas la dette effectivement accordée par le banquier. La disponibilité du crédit moyenne de $100 \%$ démontre que, sur l'ensemble de l'échantillon, on ne peut pas parler de rationnement ex post du crédit, ou de surévaluation des besoins lors de l'installation, ni de sous-estimation des besoins de financement lors de l'installation.

\section{Les termes du contrat comme déterminants du monitoring}

Les faibles corrélations entre les variables du contrat bancaire rendent non pertinente la recherche de structure sous-jacente de combinaison des termes (annexe 1). Par contre, le rôle des termes du contrat dans le monitoring peut être mis en évidence par une régression de notre indicateur de l'effort de monitoring sur les termes du contrat bancaire (tableau 2). L'équation de spécification de la régression s'écrit de la façon suivante :

$M=\beta_{1} G a r+\beta_{2} D i s p+\beta_{3} D C T+\beta_{4} C O N T+\mu(1)$

$M$ : variable binaire égale à 1 si le banquier conserve l'information comptable dans le dossier de suivi et 0 sinon,

Gar. : les garanties qui se déclinent en garanties hypothécaires, cautions et nantissements,

Disp. : la disponibilité du crédit ex ante et ex post,

$D C T$ : la dette à court terme et l'ouverture de crédit,

Contr. : les variables de contrôle comprenant la trésorerie au moment de l'installation, le chiffre d'affaires et la durée de la relation bancaire avant l'installation.

La disponibilité du crédit ex ante augmente la probabilité que le banquier conserve les états financiers dans les dossiers de suivi des entrepreneurs. Cet effet est cependant atténué lorsque la variable de contrôle « taille » est ajoutée à la régression, ce qui démontre qu'une partie de cet effet s'explique par la corrélation entre cette variable et la taille de l'entreprise. Notons toutefois que la multicolinéarité n'atteint pas des niveaux préjudiciables à la qualité de la régression - l'indice VIF moyen est de 2,33. De même, la disponibilité ex post du crédit apparaît comme un déterminant $\mathrm{du}$ monitoring. Ces résultats confirment l'hypothèse H1a : la disponibilité du crédit est un déterminant du monitoring.

Accorder des garanties hypothécaires n'a pas d'impact sur la probabilité que la banque conserve les données comptables en agence. Par contre, le taux de couverture par des cautions à un impact significatif. Ce résultat rappelle ce qu'Ono et Uesugi (2005) ont observé pour des firmes 
Tableau 2. L'effort de monitoring selon les termes du contrat bancaire

\begin{tabular}{l|lll} 
& Effort de monitoring & & \\
\hline & Sans variables & Contrôle de la taille & Contrôle de la taille et \\
de contrôle & de l'entreprise & 1.004208 \\
Hyp. & 1.004822 & 1.005469 & $1.010874^{* *}$ \\
Caut. & $1.013642^{* * *}$ & $1.011124^{* *}$ & 1.008583 \\
Nant. & 1.010327 & 1.009596 & $1.01244^{*}$ \\
Disp. ex ante & $1.014939^{* *}$ & $1.010622^{*}$ & $1.005884^{* *}$ \\
Disp. ex post & $1.006556^{* * *}$ & $1.005855^{* *}$ & $1.02901^{* * *}$ \\
DCT & $1.033262^{* * *}$ & $1.02791^{* *}$ & .9907168 \\
OC & .9987591 & .9926784 & $1.08435^{* * *}$ \\
Durée & $1.087606^{* * *}$ & $1.075748^{* *}$ & $1.646829^{*}$ \\
Trés. & & & $1.003161^{*}$ \\
CA & & $1.003909^{* *}$ & 225 \\
Number of obs. & 228 & 228 & 47.89 \\
LR chi2 (9) & 41.28 & 47.24 & 0.0000 \\
Prob. $>$ chi2 & 0.0000 & 0.0000 & 0.1537 \\
R2 ajusté & 0.1307 & 0.1495 & $67,11 \%$ \\
\hline Taux de concordance & $65,35 \%$ & $67,54 \%$ &
\end{tabular}

Note : ${ }^{*}{ }^{* *},{ }^{* *}$ significatifs à $1 \%, 5 \%$ et $10 \%$ respectivement.

L'effort de monitoring, qui est la variable expliquée, est appréhendé par la collecte et la conservation des données comptables dans le dossier de suivi des entrepreneurs. C'est une variable binaire égale à 1 si les dossiers comportent ces données et égale à 0 si ce n'est pas le cas. De ce fait, le modèle est un modèle logistique. Les variables explicatives sont les termes du contrat bancaire (garanties, disponibilité du crédit, dette à court terme, ouverture de crédit). Un coefficient de régression supérieur à 1 indique un impact favorable des termes du contrat sur la probabilité d'établir un contrat avec monitoring (suivi de l'entrepreneur). Un coefficient inférieur à 1 indique un impact défavorable.

Source : l'auteur.

japonaises : demander des garanties est un moyen, pour le banquier, d'acquérir de l'information sur l'entreprise (confirmation de l'hypothèse $\mathrm{H} 1 \mathrm{~b}$ ).

La part de la dette à court terme dans la dette totale augmente la probabilité que le banquier ait l'intention d'exercer un monitoring, et cet effet n'est pas annihilé par l'introduction d'une variable de contrôle du risque ex ante (la trésorerie au moment de l'installation), ce qui confirme l'hypothèse $\mathrm{H} 1 \mathrm{c}$ : la dette à court terme apparaît comme un instrument du monitoring.

Enfin, la durée de la relation bancaire préexistante à l'installation joue de façon significative sur le monitoring. On peut supposer que la connaissance préalable de l'entrepreneur réduit l'effort à réaliser pour mettre en place un contrat avec monitoring. D'une certaine façon, ce résultat confirme le résultat de Berger et al. (2009) qui montre que le banquier intègre les différentes sources d'information, que celleci soit hard (données comptables) ou soft (connaissance préexistante de l'entrepreneur), quand il base ses décisions de financement sur du monitoring.

\section{Propriétés incitatives du contrat et monitoring}

L'analyse a ici pour objectif de déterminer le rôle de chacun des termes du contrat dans le montant des prélèvements réalisés par l'entrepreneur. L'analyse multivariée 
permet de dégager la contribution de chacun de ces termes, indépendamment de leurs interactions avec les autres variables explicatives du revenu. L'introduction de variables d'interaction permet en outre d'identifier la contribution de chacun de ces termes selon le degré de monitoring (faible ou fort). Nous utiliserons deux proxys du monitoring : la première repose sur le principe que l'effort de monitoring est une variable dichotomique égale à 1 si la banque a conservé les données comptables et 0 sinon ; la seconde repose sur le principe que l'effort de monitoring n'est pas forcément dichotomique et qu'une catégorisation plus fine devrait permettre d'obtenir des meilleurs résultats. Dans ce but, nous construisons un indice de l'effort de monitoring à partir de la régression dans la partie précédente. La méthode de construction est explicitée dans l'annexe II. À partir de l'indice, nous distinguons ensuite les contrats supposant un monitoring fort - tiercile supérieur de l'indice - de ceux supposant un monitoring faible - tiercile inférieur de l'indice. Le tiercile intermédiaire correspond aux entreprises dont les caractéristiques des contrats ne permettent pas de prédire s'il y a monitoring ou non. L'équation de spécification s'écrit de la manière suivante :

$$
\begin{aligned}
& \operatorname{Re} v=\beta_{0}+\beta_{1} X+\beta_{2} X * M+\beta_{4} C O N T+\mu \\
& \text { Def }=\beta_{0}+\beta_{1} X+\beta_{2} X * M+\beta_{4} C O N T+\mu
\end{aligned}
$$

Rev. : le revenu de l'entrepreneur, mesuré par les flux créditeurs sur le compte personnel,

Déf. : la défaillance de l'entrepreneur (mesurée par le retard de remboursement),

$X$ : les termes du contrat bancaire,

$X^{*} M$ : les termes d'interaction des termes du contrat bancaire et du monitoring,

CON : les variables de contrôle comprenant les proxys des contraintes de revenu du ménage.
Les déterminants du revenu sont analysés par la réalisation d'une régression tobit car le revenu est une variable censurée à zéro. Les déterminants de la défaillance, variable binaire, sont analysés par la réalisation d'une régression logit. Les variables d'interaction sont construites en multipliant les variables du contrat par les variables binaires indiquant un monitoring plus ou moins fort selon les deux méthodes de catégorisation proposées ci-dessus.

Les résultats de cette première régression (tableau 3) montrent que la disponibilité du crédit détermine positivement le revenu de l'entrepreneur quand il y a monitoring. Sinon, celle-ci n'a pas d'impact sur le revenu, ce qui confirme l'hypothèse H2a : la disponibilité du crédit ex post est un facteur clé de la performance financière des entreprises financées via un contrat avec monitoring.

Les garanties hypothécaires ont un effet négatif sur le revenu hormis pour les entreprises financées via un contrat sans monitoring (rappelons que le niveau d'endettement est contrôlé par la variable DMLT/ $f(u x)$. Les autres types de garanties n'ont pas d'incidence sur le revenu de l'entrepreneur. L'hypothèse $\mathrm{H} 2 \mathrm{~d}$ peut expliquer ce résultat : la moindre vigilance du banquier aboutit à des choix d'investissement risqués qui ont pour conséquence une chute de revenu des entrepreneurs.

La dette à court terme a un effet positif sur le revenu des entrepreneurs financés via un contrat sans monitoring uniquement dans le cas où le proxy utilisé est l'indice. De notre point de vue, il rend compte du revenu d'un faible nombre d'entrepreneurs dont le contrat ne comporte a priori pas de dette à court terme. Il nous paraît difficile de différencier l'effet direct de cet apport de fonds sur la trésorerie de l'entrepreneur et un potentiel effet incitatif à ce stade de l'analyse. 
Tableau 3. Impact des termes du contrat bancaire sur le revenu

\begin{tabular}{|c|c|c|c|}
\hline & \multicolumn{3}{|c|}{ Revenu } \\
\hline & $\begin{array}{l}\text { Sans prendre en compte } \\
\text { le monitoring } \\
\text { (Modèle 1) }\end{array}$ & $\begin{array}{c}\text { En prenant en compte le } \\
\text { monitoring par la présence } \\
\text { de documents comptables } \\
\text { (Modèle 2) }\end{array}$ & $\begin{array}{c}\text { En prenant en compte } \\
\text { le monitoring par l'indice } \\
\text { (Modèle 3) }\end{array}$ \\
\hline Disp. ex ante & 11.50916 & 10.5048 & $16.75911^{*}$ \\
\hline Disp. ex post & $.0599004^{*}$ & $.0992565 * *$ & $.0684232 * *$ \\
\hline $\begin{array}{l}\text { Disp. ex post * Monit. } \\
\text { Faible }\end{array}$ & & $-.0834801 *$ & -.0274565 \\
\hline Hyp. & -.1996621 ** & $-.2896668^{*}$ & $-.2596621 * *$ \\
\hline Hyp. * Monit. Faible & & .1607219 & .1548116 \\
\hline Caut. & 1.075373 & -2.472873 & -1.074336 \\
\hline Caut. * Monit. Fort & & 5.01147 & 7.12686 \\
\hline Nant. & -3.284685 & -1.478972 & -3.221423 \\
\hline DCT/flux & -.2051647 & -.2271454 & -.2184429 \\
\hline DCT/flux * Monit. Faible & & .0614975 & $5.548335^{* *}$ \\
\hline OC/flux & -71.47582 & -.7277988 & -72.40729 \\
\hline DMLT/flux & 20.86693 & 16.79808 & 30.6788 \\
\hline Stock PF/Prod. & $-15.46497 * * *$ & $-12.55178 * *$ & $-11.21362 * *$ \\
\hline Age & $1.607847 * * *$ & $1.712901 * * *$ & $1.112802^{* *}$ \\
\hline Age * conjoint salarié & $-.4455002 * *$ & $-.4528473 * *$ & $-.379645^{* *}$ \\
\hline Valorisation & $.0227861 * * *$ & $.0212193 * *$ & $.0239131 * * *$ \\
\hline cons & -21.75894 & -24.05381 & -16.65172 \\
\hline Number of obs. & 143 & 143 & 139 \\
\hline LR chi2 & (12) 42.98 & (16) 46.02 & (16) 48.48 \\
\hline Prob. > chi2 & 0.0000 & 0.0001 & 0.0000 \\
\hline R2 ajusté & 0.0318 & 0.0341 & 0.0370 \\
\hline
\end{tabular}

Note : Le revenu est une variable censurée à 0 . Pour cette raison, la régression est un modèle tobit. Les variables d'interaction permettent d'identifier le rôle de chacun des termes du contrat selon l'intensité du monitoring bancaire. Les garanties hypothécaires ont un impact négatif sur le revenu des entrepreneurs sauf quand le monitoring bancaire est faible. La dette à court terme n'a pas d'impact significatif sur le revenu sauf quand le monitoring est faible. Dans ce cas, la dette à court terme implique un revenu supérieur.

Source : l'auteur.

L'âge de l'entrepreneur a un impact significatif sur le revenu : plus l'entrepreneur est âgé, plus le revenu augmente. L'introduction de la variable d'interaction âge/activité salariée du conjoint montre que cette relation s'explique davantage par une augmentation de la contrainte de revenu avec l'âge (autonomie du ménage et enfants) plutôt que par une meilleure performance des entrepreneurs plus matures. Les entrepreneurs auraient tendance à moins prélever quand leurs conjoints apportent un revenu supplémentaire. Cette hypothèse est confirmée par l'analyse de la défaillance (tableau 4) : l'âge est un facteur de risque de défaillance, ce qui laisse supposer que l'augmentation de revenu associée à l'âge est contrainte.

L'analyse de la défaillance complète celle du revenu. D'après les modèles de régression 4 à 6 (tableau 4), la disponibilité du crédit n'a pas d'influence sur le retard de remboursement. En revanche, les garanties hypothécaires impliquent plus de risque de 
Tableau 4. Impact des termes du contrat bancaire sur le risque de défaillance

\begin{tabular}{|c|c|c|c|}
\hline & \multicolumn{3}{|c|}{ Retard de remboursement } \\
\hline & $\begin{array}{l}\text { Sans prendre en } \\
\text { compte la dimension } \\
\text { relationnelle } \\
\text { (Modèle 4) }\end{array}$ & $\begin{array}{c}\text { En prenant en compte la dimen- } \\
\text { sion relationnelle par la présence } \\
\text { de documents comptables } \\
\text { (Modèle 5) }\end{array}$ & $\begin{array}{c}\text { En prenant en compte } \\
\text { la dimension relationnelle } \\
\text { par l'indice } \\
\text { (Modèle 6) }\end{array}$ \\
\hline Dispo. ex ante & .4074673 & .4023633 & \begin{tabular}{|l|}
.7236798 \\
\end{tabular} \\
\hline Dispo. ex post & -.003354 & -.0020889 & -.0063303 \\
\hline $\begin{array}{l}\text { Dispo. ex post * } \\
\text { monitoring fort }\end{array}$ & & -.003474 & .0055667 \\
\hline Hyp. & $.0140089 *$ & $.02051 * *$ & $.0215888^{* *}$ \\
\hline $\begin{array}{l}\text { Hyp. * } \\
\text { monitoring faible }\end{array}$ & & -.0116108 & -.0104799 \\
\hline Caut. & .0868979 & .0900218 & .703118 \\
\hline Nant. & .2889424 & .1505753 & .5927407 \\
\hline DCT/flux & $.0510772 * * *$ & .0426983 & $.2610352 * * *$ \\
\hline $\begin{array}{l}D C T / f l u x \text { * } \\
\text { monitoring fort }\end{array}$ & & .0060636 & $-.1911924 * *$ \\
\hline OC/flux & .0387292 & .0285157 & $.1347966 * * *$ \\
\hline $\begin{array}{l}\text { OClflux* } \\
\text { monitoring fort }\end{array}$ & & .0452088 & $-.2555898 * *$ \\
\hline DMLT/flux & .2590999 & .3085377 & .5132071 \\
\hline Age & $.1210302 * * *$ & $.1172307 * * *$ & $.1624733 * * *$ \\
\hline Age * conjoint salarié & -.0082738 & -.0095678 & $-.0290042 *$ \\
\hline _cons & $-5.883618^{* * *}$ & $-5.746627 * * *$ & $-7.738037 * * *$ \\
\hline Number of obs. & 229 & 229 & 224 \\
\hline LR chi2 & (10) 35.73 & (14) 37.32 & (14) 56.02 \\
\hline Prob. > chi2 & 0.0001 & 0.0007 & 0.0000 \\
\hline Pseudo R2 & 0.1684 & 0.1759 & 0.2704 \\
\hline
\end{tabular}

Note : Le retard de remboursement est une variable binaire. Le modèle est un modèle logit. Les variables d'interaction permettent d'identifier le rôle de chacun des termes du contrat selon l'intensité du monitoring bancaire. On constate par exemple que les garanties hypothécaires ont globalement un effet positif (mais faiblement significatif) sur le risque de défaillance. L'introduction de la variable d'interaction montre que cet effet est nul pour les entreprises financées via un contrat sans monitoring.

Source : l'auteur.

défaillance mais pas pour les entreprises financées via un contrat sans monitoring. Ce résultat confirme l'hypothèse selon laquelle les garanties réduisent l'effort de monitoring et implique ainsi une prise de risque supplémentaire $(\mathrm{H} 2 \mathrm{~d})$ quand la relation bancaire est censée être forte.

L'impact de la dette à court terme varie selon que l'on considère les entrepreneurs financés via un contrat avec ou sans monitoring (modèle 6). La dette à court terme tend à réduire la probabilité de défaillance lorsqu'il y a monitoring, ce qui est conforme à la théorie de l'agence : la dette à court terme est un mode de financement qui structure le monitoring et discipline le dirigeant. Lorsqu'il n'y a pas monitoring, la dette à court terme est associée à plus de défaillance, ce qui proviendrait d'une utilisation de la dette à court terme en dernier 
recours, lorsque le montant des revenus atteint un seuil minimum. Cette interprétation expliquerait le résultat qui précède, mettant en évidence un impact positif de la dette à court terme sur le revenu de l'entrepreneur.

\section{$*$}

$* *$

Cet article contribue à la recherche sur la relation banque/entreprise de deux façons : d'abord en identifiant le rôle des termes du contrat bancaire dans le monitoring bancaire puis en étudiant l'impact du monitoring sur les propriétés incitatives des termes du contrat.

L'analyse empirique menée sur l'installation en viticulture confirme le rôle de certains termes du contrat bancaire dans la mise en place du monitoring. Les cautions sont étroitement associées au monitoring bancaire, sans doute du fait de l'information qu'elle procure au banquier sur le patrimoine de l'entrepreneur et des personnes prêtes à soutenir le projet. La dette à court terme est aussi fortement liée au monitoring, au point de n'être que très rarement utilisée pour le financement des entrepreneurs susceptibles d'être financés via un contrat sans monitoring. Pour ces entrepreneurs, les besoins à court terme sont financés par de l'ouverture de crédit, qui a pour avantage d'être limité et de ne pas provoquer de renégociation qui comporte des coûts de transaction.

La deuxième partie de l'analyse empirique permet d'identifier les propriétés incitatives des termes du contrat bancaire selon qu'il y ait monitoring ou non. La disponibilité du crédit ex post pour les entrepreneurs financés via un contrat avec monitoring a un impact positif sur la performance en termes de revenu. En revanche, une meilleure disponibilité du crédit n'implique pas forcément un risque de défaillance moindre. Ces résultats démontrent que la banque ne suit pas une logique uniquement prudentielle mais qu'elle accompagne l'entrepreneur dans le développement de l'entreprise. La qualité du monitoring peut toutefois être altérée par les garanties hypothécaires, qui impliquent plus d'échecs à la fois en termes de revenus pour l'entrepreneur et de défaillance.

La recherche comporte certaines limites. Si l'homogénéité de l'échantillon évite les biais associés à la fois aux variables sectorielles et de cycle de vie (l'analyse se concentre sur le cas de la reprise et de la création d'entreprise), elle réduit le pouvoir de généralisation de l'étude. D'autre part, l'étude gagnerait sans doute à être réalisée sur un échantillon plus large et sur des données de panel. Il faudrait alors trouver un indicateur de la dimension relationnelle autre que celui utilisé dans notre étude, qui est très spécifique au contexte de collecte des données et au secteur étudié. Le nombre de rendez-vous, d'e-mail ou d'entretiens téléphoniques entre les chargés d'affaires et les entrepreneurs ou encore un questionnaire adressé aux chargés d'affaires ou aux entreprises pourraient remplir ce rôle.

Au-delà des limites méthodologiques notre analyse montre que le monitoring bancaire peut être appréhendé via les caractéristiques des termes du contrat. Pour les banques, une caractérisation du monitoring peut être un outil de management utile pour optimiser la répartition des chargés d'affaires selon les entreprises et les secteurs. Ce pourrait être aussi un moyen de rendre compte d'une des dimensions essentielles de leur rôle dans le développement économique. 


\section{RÉFÉRENCES BIBLIOGRAPHIQUES}

Aghion P., Bolton P. (1992). An Incomplete Contracts Approach to Financial Contracting. The Review of Economic Studies, vol. 59, p. 473-494.

Akerlof G. A. (1970). The Market for 'Lemons': Quality Uncertainty and the Market Mechanism. Quarterly Journal of Economics, vol. 84, $\mathrm{n}^{\circ} 3$, p. 488-500.

Barry P. J., Robison L. J. (2001). Agricultural Finance: Credit, Credit Constraints, and Consequences. In Gardner B., Rausser G. (dir.), « Handbook of Agricultural Economics », Elsevier Science B.V.

Berger A. N., Klapper L. F., Udell G. F. (2001). The Ability of Banks to Lend to Informationally Opaque Businesses. Journal of Banking and Finance, vol. 25, p. 2127-2167.

Berger A. N., Frame W. S., Miller N. (2005). Credit Scoring and the Availability, Price, and Risk of Small Business Credit. Journal of Money, Credit, and Banking, $\mathrm{n}^{\circ} 37$, p. 191-222.

Berger A. N., Cowan A. M., Frame W. S. (2009). The Surprising Use of Credit Scoring in Small Business Lending by Community Banks and the Attendant Effects on Credit Availability and Risk. Working Paper 2009-9, Federal Reserve Bank of Atlanta.

Berger A. N., Udell G. F. (1995). Relationship Lending and Lines of Credit in Small Firm Finance. The Journal of Business, vol. 68, p. 351-381.

Bester H. (1996). The Role of Collateral in a Model of Debt Renegotiation. Journal of Money, Credit, and Banking, vol. 26, p. 72-86.

Bolton P., Sharfstein D. S. (1990). A Theory of Predation Based on Agency Problems in Financial Contracting. American Economic Review, vol. 80, p. 94-106.

Boot A. W. A., Thakor A. V. (1994). Moral Hazard and Secured Lending in an Infinitely Repeated Credit Market Game. International Economic Review, vol. 35, p. 899-920.

Chakraborty A, Hu C. X. (2006). Lending Relationships in Line-of-Credit and Nonline-ofCredit loans: Evidence from Collateral Use in Small Business. Journal of Financial Intermediation, vol. 15, p. 86-107.

Chassard M., Chevalier B. (2007). Un large éventail de revenus agricoles. Agreste, cahier $\mathrm{n}^{\circ} 2$, juillet.

Cole R. A. (1998). The Importance of Relationships to the Availability of Credit. Journal of Banking \& Finance, vol. 22, p. 959-977.

Degryse H., Van Cayseele P. (2000). Relationship Lending within a Bank-Based System: Evidence from Small Business Data. Journal of Financial Intermediation, vol. 9, p. 90-109.

Elsas R. (2005). Empirical determinants of relationship lending. Journal of Financial Intermediation, vol. 14, p. 32-57.

Fama E. (1985). What's different about banks? Journal of Monetary Economics, vol. 15, p. 29-36.

Flannery M. J. (1986). Asymmetric Information and Risky Debt Maturity Choice. The Journal of Finance, vol. 41, p. 19-37.

Jensen M. C. (1986). Agency Costs of Free Cash Flow, Corporate Finance, and Takeovers. The American Economic Review, vol. 76, p. 323-329.

Kano M., Hirofumi U., Udell G. F., Watanabe W. (2010). Information Verifiability, Bank Organization, Bank Competition and BankBorrower Relationships. Journal of Banking and Finance, vol. $35, \mathrm{n}^{\circ} 5$.

Manove M. A., Padilla J., Pagano M. (2001). Collateral vs. Project Screening. Centre For Studies in Economics and Finance, Working Paper.

Milgrom P., Roberts J. (1992). Economics, Organizations and Management. Englewood Cliffs, N. J.: Prentice Hall.

Ono A., Uesugi I. (2005). The Role of Collateral and Personal Guarantees in Relationship Lending: Evidence from Japan's Small Business Loan Market. RIETI Discussion Paper Series 05-E-027.

Petersen M. A. (2004). Information: Hard and Soft. NBER working paper. 
Petersen M. A., Rajan R. G. (1994). The Benefits of Lending Relationships: Evidence from Small Business Data. The Journal of Finance, vol. 49, p. 3-37.

Rajan R., Winton A. (1995). Covenants and Collateral as Incentives to Monitor. The Journal of Finance, vol. 50, p. 1113-46.

Rougès V. (2007). Les informations comptables répondent-elles aux besoins informationnels des banquiers lors de l'octroi de crédit à une entreprise ? Communication au Congrès de l'Association Française de Comptabilité 2007.

Stein J. C. (2002). Information Production and Capital Allocation: Decentralized versus Hierarchical Firms. The Journal of Finance, vol. 57, p. 1891-1921.

Stiglitz J. E., Weiss A. (1981). Credit Rationing in Markets with Imperfect Information. American Economic Review, vol. 71, $\mathrm{n}^{\circ} 3$, p. 393-410.

\section{ANNEXES}

\section{Annexe 1. Matrice de corrélations}

Les corrélations entre les différentes variables, bien que significatives, sont assez faibles (aucune n'est supérieure à 0,5 ), ce qui rend inopérante toute démarche de factorisation ${ }^{6}$.

\section{Annexe 2. L'effort de monitoring, de la variable binaire à l'indice}

La régression logistique expliquant l'intention de monitoring du banquier peut être utilisée pour établir un indice à partir de ses coefficients et des valeurs observées des variables explicatives. Autrement dit, nous construisons une variable continue que l'on nomme «monitoring » à partir des valeurs prédites par la régression sur notre échantillon. Celle-ci est comprise entre 0 et 1 . Nous l'exprimons en \%. L'indice peut être considéré comme un indicateur de la dimension relationnelle.

Tableau 5. Corrélations entre les termes du contrat bancaire

\begin{tabular}{l|c|c|c|c|c|c|c|c|c} 
& CA & Rev. & $\begin{array}{c}\text { Disp. ex } \\
\text { ante }\end{array}$ & $\begin{array}{c}\text { Disp. ex } \\
\text { post }\end{array}$ & Hyp. & Caut. & Nant. & DCT & OC \\
\hline CA & 1.0000 & & & & & & & & \\
\hline Rev. & $0.2857^{*}$ & 1.0000 & & & & & & & \\
\hline Disp. ex ante & $0.2391^{*}$ & 0.1405 & 1.0000 & & & & & & \\
\hline Disp. ex post & $0.2125^{*}$ & 0.0905 & $-0.2807^{*}$ & 1.0000 & & & & & \\
\hline Hyp. & $-0.1738^{*}$ & -0.0999 & 0.1032 & 0.0709 & 1.0000 & & & & \\
\hline Caut. & $0.2429^{*}$ & 0.0408 & -0.0597 & -0.0912 & $-0.3707^{*}$ & 1.0000 & & & \\
\hline Nant. & 0.1034 & -0.0040 & 0.0462 & -0.0794 & $-0.1416^{*}$ & $-0.1908^{*}$ & 1.0000 & & \\
\hline DCT & $0.1457^{*}$ & -0.0513 & 0.0927 & $-0.2151^{*}$ & $-0.1267^{*}$ & 0.0492 & 0.0022 & 1.0000 & \\
\hline OC & 0.0767 & -0.0704 & -0.1255 & $-0.2365^{*}$ & $-0.2181^{*}$ & 0.0347 & $0.1381^{*}$ & 0.0237 & 1.0000
\end{tabular}

Note : *significativité à $5 \%$.

6. Nous avons testé la possibilité de dégager la dimension relationnelle du contrat bancaire par une analyse en composantes principales orthonormées. Cela se révèle impossible du fait de la faiblesse des corrélations, ce qui est confirmé par le calcul de l'indice de Keyser-Meyer-Olkin qui est inférieur à 0.6. 
Tableau 6. L'indice monitoring

\begin{tabular}{l|rrrrr} 
Stat. & Obs. & Moy. & Ec. type & Min & Max \\
& 230 & $49,13 \%$ & $18,15 \%$ & $12,77 \%$ & $93,96 \%$ \\
\hline Centiles & 25 & 33 & 50 & 66 & 75 \\
& $34,94 \%$ & $39,19 \%$ & $46,44 \%$ & $53,98 \%$ & $62,77 \%$
\end{tabular}

À partir de là, nous pouvons construire des classes selon la dimension relationnelle du contrat qui soient plus fines que la simple variable dichotomique basée sur la détention de l'information comptable par le banquier. Dans cette recherche, nous construisons l'indice à partir de la régression (1) du tableau 2, car elle isole les termes du contrat bancaire des variables de performances que pourraient être la taille et la trésorerie.

Les hypothèses sur les relations entre performance et variables du contrat bancaire pourront être testées sur chacune de ces classes. Nos données nous permettent d'accéder aux performances observables à partir des comptes bancaires : le revenu de l'entrepreneur, mesuré par les flux sur le compte personnel et le retard de remboursement. Notons que pour le banquier, ces variables de performance présentent un avantage sur les données comptables, celles d'être immédiates alors que les données comptables informent le banquier bien plus tardivement (Rougès, 2007). De plus, les données comptables dont nous disposons concernent le plus souvent les exercices suivant l'installation (entre 1998 et 2003 tandis que les données bancaires sont de 2005). En cela, elles ne sont pas révélatrices des performances ex post de l'installation, mais plutôt du processus d'investissement suivant l'installation.

\section{Annexe 3. Analyse bivariée}

Le tableau 8 montre que la disponibilité du crédit est corrélée positivement avec le revenu pour les entreprises pour lesquelles la dimension relationnelle du contrat bancaire est forte, que ce soit ex ante ou ex post. A contrario, on ne constate aucune corrélation entre disponibilité du crédit et revenu pour les entreprises financées via un contrat sans monitoring.

Tableau 7. Corrélation entre le revenu et les termes du contrat bancaire selon l'intensité du monitoring

\begin{tabular}{l|c|c|c|c|c|c|c|c|c} 
& \multicolumn{1}{|c}{ Termes du contrat } \\
\hline Monitoring... & $\begin{array}{c}\text { Disp. ex } \\
\text { ante }\end{array}$ & $\begin{array}{c}\text { Disp. ex } \\
\text { post }\end{array}$ & Hyp. & Caut. & Nant. & DCT & $\begin{array}{c}\text { DCTI } \\
\text { flux }\end{array}$ & OC & $\begin{array}{c}\text { OCI } \\
\text { flux }\end{array}$ \\
\hline \multicolumn{10}{c}{ Dossiers avec ou sans comptabilité } \\
\hline ... sans compta & 0.06 & -0.08 & -0.00 & -0.05 & 0.01 & 0.00 & -0.01 & -0.08 & $-0.16^{*}$ \\
$\ldots$ avec compta & $0.18^{*}$ & $0.20^{*}$ & $-0.19^{*}$ & 0.11 & -0.03 & -0.12 & -0.15 & -0.04 & -0.09 \\
\hline \multicolumn{10}{c}{ Classement en tierciles déterminés à partir de l'indice } \\
\hline$\ldots$ faible & 0.21 & -0.05 & 0.16 & -0.04 & -0.04 & 0.02 & $0.25^{*}$ & -0.13 & -0.19 \\
$\ldots$ moyen & $-0.27^{*}$ & 0.03 & $-0.27^{*}$ & 0.02 & 0.06 & $0.33^{* *}$ & -0.18 & 0.16 & -0.24 \\
$\ldots$ fort & $0.25^{* *}$ & $0.25^{* *}$ & -0.10 & 0.03 & -0.02 & -0.18 & $-0.25^{*}$ & -0.13 & -0.10 \\
\hline Ensemble & $0.14^{*}$ & 0.09 & -0.10 & 0.04 & -0.00 & -0.05 & -0.07 & 0.14 & -0.13
\end{tabular}

Note : $\left({ }^{*}\right),\left(^{* *}\right)$ test des corrélations de Pearson, significatif à $10 \%$ et $5 \%$, respectivement. 
Tableau 8. Les termes du contrat bancaire selon l'intensité du monitoring et le retard de remboursement

\begin{tabular}{|c|c|c|c|c|c|c|c|c|c|c|}
\hline Monitoring... & \begin{tabular}{|c|} 
Retard de \\
remb.
\end{tabular} & $\begin{array}{l}\text { Disp. ex } \\
\text { ante }\end{array}$ & $\begin{array}{c}\text { Disp. ex } \\
\text { post }\end{array}$ & Hyp. & Caut. & Nant. & $D C T$ & $\begin{array}{l}\text { DCTI } \\
\text { flux }\end{array}$ & $O C$ & $\begin{array}{l}\text { OCl } \\
\text { flux }\end{array}$ \\
\hline \multicolumn{11}{|c|}{ Dossiers avec ou sans comptabilité } \\
\hline \multirow{4}{*}{ Sans compta } & & & & $(*)$ & & & $(* *)$ & $(* * *)$ & & $(*)$ \\
\hline & Non & 62,64 & 89,97 & 15,64 & 29,17 & 11,62 & 3,90 & 1,31 & 9,62 & 3,17 \\
\hline & Oui & 69,45 & 90,80 & 25,75 & 30,51 & 7,55 & 11,89 & 5,88 & 5,89 & 5,35 \\
\hline & Total & 63,50 & 90,08 & 17,12 & 29,37 & 11,02 & 5,05 & 1,91 & 9,06 & 3,48 \\
\hline \multirow{4}{*}{ Avec compta } & & & $(* *)$ & $(* *)$ & & & $(* * *)$ & $(* * *)$ & & $(*)$ \\
\hline & Non & 70,28 & 110,51 & 13,13 & 44,98 & 15,08 & 8,12 & 4,98 & 8,92 & 3,46 \\
\hline & Oui & 75,83 & 80,95 & 25,32 & 37,55 & 17,15 & 20,25 & 16,60 & 7,58 & 5,02 \\
\hline & Total & 71,50 & 104,20 & 15,82 & 43,34 & 15,53 & 10,69 & 7,42 & 8,64 & 3,79 \\
\hline \multicolumn{11}{|c|}{ Classement en tierciles déterminés à partir de l'indice } \\
\hline \multirow{3}{*}{... faible (f) } & Non & 56,28 & 82,58 & 15,30 & 21,19 & 9,28 & 0,85 & 0,28 & 4,72 & 2,15 \\
\hline & Oui & 63,43 & 76,62 & 29,72 & 22,46 & 7,69 & 1,05 & 0,74 & 6,21 & 6,40 \\
\hline & Total & 56,93 & 82,03 & 16,61 & 21,31 & 9,14 & 0,87 & 0,32 & 4,86 & 2,53 \\
\hline \multirow{4}{*}{... moyen } & & & & $(* * *)$ & $(* * *)$ & & & $(* * *)$ & & $(* *)$ \\
\hline & Non & 65,40 & 117,27 & 15,40 & 50,41 & 11,89 & 1,39 & 0,63 & 8,31 & 3,52 \\
\hline & Oui & 69,14 & 97,67 & 34,35 & 18,47 & 20,73 & 8,54 & 10,56 & 9,30 & 6,28 \\
\hline & Total & 66,20 & 113,09 & 19,44 & 43,60 & 13,78 & 2,91 & 2,77 & 8,52 & 4,13 \\
\hline \multirow{4}{*}{... fort } & & & & & & & $(* *)$ & $(* * *)$ & & \\
\hline & Non & 77,65 & 120,51 & 13,14 & 46,57 & 21,39 & 14,84 & 8,69 & 8,05 & 4,75 \\
\hline & Oui & 79,91 & 79,54 & 18,60 & 54,29 & 10,53 & 27,13 & 19,87 & 5,25 & 3,65 \\
\hline & Total & 78,17 & 111,35 & 14,40 & 48,36 & 18,89 & 17,68 & 11,16 & 7,40 & 2,15 \\
\hline & & & & & & & & & & \\
\hline \multirow{4}{*}{ Ensemble } & & $\left({ }^{*}\right)$ & & $(* *)$ & & & $(* * *)$ & $(* * *)$ & & $(* *)$ \\
\hline & Non & 65,90 & 99,10 & 14,53 & 36,17 & 13,15 & 5,81 & 2,94 & 9,30 & 3,30 \\
\hline & Oui & 73,22 & 85,07 & 25,51 & 34,49 & 12,98 & 16,64 & 12,11 & 6,83 & 5,17 \\
\hline & Total & 67,13 & 96,69 & 16,52 & 35,86 & 13,12 & 7,71 & 4,48 & 8,86 & 3,63 \\
\hline
\end{tabular}

Note : $\left({ }^{*}\right),\left(^{* *}\right)\left({ }^{* *}\right)$ t-test significatif à $10 \%, 5 \%$ et $1 \%$, respectivement $(f)$ le nombre d'entreprises en retard de remboursement est trop faible pour que la différence de moyenne ait une valeur statistique.

Il existe une corrélation négative entre la part de garanties hypothécaires et le revenu pour les entreprises de la catégorie intermédiaire. Ce résultat est faiblement significatif et nécessite d'être contrôlé par une variable d'endettement.

La part de dette à court terme dans le total de l'endettement est positivement corrélée au revenu pour les entreprises bénéficiant d'un monitoring moyen. Une interprétation possible serait que la dette à court terme représente ici un privilège pour quelques entrepreneurs dans une catégorie d'entrepreneurs pour lesquels ce moyen de financement est très peu utilisé.

Les résultats sont quelque peu différents quand on rapporte la dette à court terme au chiffre d'affaires de l'entreprise : celle-ci est négativement corrélée au revenu pour les entreprises bénéficiant d'un contrat avec monitoring et positivement pour lesquelles le monitoring est le plus faible. Le premier résultat s'explique par la nécessité de conserver le cash pour le remboursement de la dette, le second par une plus grande latitude dans l'utilisation de la trésorerie pour les entrepreneurs qui ont accès au financement à court terme au sein d'une catégorie d'entrepreneurs privés de ce mode de financement. 
La relation entre le retard de remboursement et les termes du contrat bancaire est étudiée en comparant les moyennes des variables du contrat des entreprises défaillantes et des autres.

L'absence de significativité de la disponibilité du crédit ex ante et ex post sur le retard de remboursement démontre une certaine maîtrise du financement par les banques : les difficultés financières des entreprises ne s'expliquent pas par une offre de crédit à long terme trop importante de la part des banques.

En revanche, la relation fortement significative entre garanties hypothécaires et retard de remboursement rend compte d'un risque d'endettement excessif dû à la couverture que représentent les garanties hypothécaires pour les entreprises de la catégorie intermédiaire. Les garanties hypothécaires auraient pour effet d'induire un endettement excessif et de réduire l'effort de monitoring des banques, ce qui correspond à l'hypothèse H2a. La confirmation de cette hypothèse nécessite de contrôler le niveau d'endettement, ce qui est fait dans la régression logistique réalisée dans le corps de l'article. À l'inverse, les cautions impliqueraient une moindre prise de risque. Ces effets sont d'autant plus forts que la dimension relationnelle du contrat n'est pas clairement définie, ce qui expliquerait qu'ils soient constatés pour les entrepreneurs de la catégorie intermédiaire.

Enfin, la dette à court terme est clairement associée à la défaillance. Il est toutefois intéressant de constater que le niveau critique de dette à court terme associée à la défaillance dépend de l'effort monitoring bancaire : quand l'effort de monitoring est faible (pas de comptabilités dans le dossier), la part de la dette à court terme dans l'endettement est en moyenne de 3,90\% pour les entreprises non défaillantes et de $11,89 \%$ pour les entreprises défaillantes; quand l'effort de monitoring est fort (comptabilité dans le dossier), la part de la dette à court terme dans l'endettement est en moyenne de $8,12 \%$ et de $19,87 \%$, pour, respectivement, les entreprises non défaillantes et les entreprises défaillantes. Ce phénomène est confirmé lorsque l'on considère la dette à court terme par rapport aux flux sur le compte professionnel (proxy du chiffre d'affaires).

La relation entre la défaillance et l'ouverture de crédit pour les entreprises pour lesquelles le monitoring est « faible » montre que l'ouverture de crédit est le moyen de financement privilégié de la trésorerie pour ces entreprises : les entreprises dont les trésoreries sont tendues (et donc plus propices à la défaillance) présentent un niveau d'ouverture de crédit supérieur à la moyenne. 